\title{
Aspergiloma Pulmonar em Paciente Portadora de HIV:
}

\section{Relato de Caso}

\author{
Leandro Altrão Martines \\ Universidade Severino Sombra, Acadêmico de Medicina, \\ leandroaltrao@hotmail.com \\ Daniel Pio de Oliveira \\ Hospital Heliópolis, Médico Residente de Clínica Médica \\ danpio.med@gmail.com \\ Pedro Paulo Mexas \\ Universidade Severino Sombra, Professor Titular de \\ Imunologia e Professor Adjunto de Histologia da UNIRIO, \\ mexas@uol.com.br
}

Resumo: Relata-se o caso de uma paciente de 45 anos portadora de síndrome da imunodeficiência adquirida (SIDA), não aderente ao tratamento para tuberculose pulmonar, que evoluiu com hemoptise importante. Aos exames de imagem, evidenciavam-se cavernas pulmonares com presença, no interior destas, de estruturas de partes sólidas. Foram obtidos, no lavado broncoalveolar, Aspergillus sp. Devido às repetidas hemoptises, a paciente foi submetida à cirurgia. Porém, apresentou má evolução no pós-operatório e apresentou como desfecho o óbito.

Palavras-Chave: Aspergiloma pulmonar. Tuberculose. SIDA

\section{Pulmonary Aspergilloma in a Patient whith HIV: \\ a Case Report}

\begin{abstract}
We report a case of a 45 year-old female pacient that had AIDS and pulmonary tuberculosis without effective treatment who developed severe hemoptisis. At imaging, show up with pulmonary caverns presence, within these, the structures of solid parts. Were obtained by bronchoalveolar lavage, Aspergillus sp. Because of repeated hemoptysis, the patient underwent surgery. However, showed poor outcome in postoperative and presented as an outcome of death.
\end{abstract}

Keywords: Pulmonary aspergilloma. Tuberculosis. AIDS 
Medicina

\section{Introdução}

A aspergilose pulmonar pode ser classificada em quatro tipos: aspergilose pulmonar invasiva, aspergilose semi-invasiva, aspergilose broncopulmonar alérgica e o aspergiloma pulmonar [Baradkar et al, 2009].

Aspergiloma ou micetoma é definido como a presença de uma massa de micélio fúngico, células inflamatórias, fibrina, muco e debris numa cavidade pulmonar pré-existente [Soubani \& Chandrasekar, 2002]. O agente etiológico desta enfermidade é um fungo Aspergillus sp. e esta é considerada a forma mais comum de acometimento pulmonar desse patógeno. Podem ser divididos em simples, quando o aspergiloma se desenvolve em cavidades circundadas por tecido pulmonar ou pleural normais, ou complexos [Grima et al, 2009].

O aspergiloma ocorre em 10\%-15\% das doenças cavitárias pulmonares. Usualmente acomete cavidades decorrentes de tuberculose pulmonar [Park \& Theon, 2002]. Entretanto, diversas enfermidades já foram relacionadas como a pneumonia por Klebsiella, sarcoidose, cistos broncogênicos, enfisema bolhoso, bronquiectasias, abscessos curados, ente outros.

A ocorrência dessa lesão em pacientes soropositivos para HIV já foi relatada pela literatura.

Descreve-se aqui o caso de uma paciente portadora de SIDA, com sequelas de tuberculose que desenvolveu aspergiloma pulmonar.

\section{Relato}

Paciente V. L. S. C., 45 anos, sexo feminino, cor parda, natural de Fortaleza / Ceará e residente na cidade de São Paulo, veio transferida de outro hospital com queixa de tosse produtiva há 9 meses com escarro de aspecto amarelado.

Referiu ainda perda $10 \mathrm{Kg}$ nesse período e febre vespertina não aferida. Relatou que, há dois dias da admissão, passou a apresentar hemoptise.

Apresentava como antecedentes ser soropositiva para HIV há 9 anos, tendo abandonado o tratamento a um ano. Trouxe relatório que constava ter feito uso, como terapia antiretroviral, (d4T + ddI + IDV), entre 2000 e 2002 com abandono do tratamento por intolerância gástrica. Entre 2003 e 2008 (um ano antes da admissão em nosso serviço), fez uso de TDF + 3TC + EFV também tendo abandonado o tratamento. Constavam os seguintes exames: CD4 de 240 células $/ \mu \mathrm{L}, \mathrm{CD} 8$ de 1.652 células $/ \mu \mathrm{L}$ e carga viral de 179.968 datados de 10 meses antes do internamento.

Ainda como história prévia, possuía passado de tuberculose em 2000 e em 2005, com abandono do tratamento cinco meses antes da admissão, além de anemia secundária ao HIV, papulose bowenóide, lesão cervical de alto grau e cor anêmico quatro meses antes da internação por hemoptise.

Durante internação no outro serviço, a paciente apresentou icterícia obstrutiva por coledocolitíase, anemia grave (Hemoglobina $<5,4 \mathrm{mg} / \mathrm{dL}$ ) e endoscopia digestiva alta com lesão ulcerada única. 
Em nosso serviço, a paciente deu entrada com icterícia leve, eupnéica com presença, à ausculta pulmonar, de estertores crepitantes na base do hemitórax direito.

À radiografia de tórax da admissão, foram detectadas duas regiões hiperdensas com sinal do ar crescente nos lobos superiores bilateralmente (Figura 1.)

A paciente realizou três coletas de escarro com pesquisa negativa para BAAR, sendo verificados apenas pseudomicélios e blastoconídeos sugestivos de Candida $s p$.

Foi submetida à endoscopia digestiva alta que evidenciou esofagite erosiva e presença de placas brancas no esôfago sugestivas de monilíase esofágica. A ultrassonografia de abdome revelou hepatomegalia discreta e sinais de colecistopatia calculosa.

A tomografia de tórax revelou a presença de caverna pulmonar com densidade de partes moles em seu interior (Figura 2).

A paciente foi submetida à broncofibroscopia que identificou conteúdo sanguinolento na traquéia, pulmão direito e óstio brônquico de língula de pulmão esquerdo.

Foi realizado lavado broncoalveolar que foi negativo para BAAR, porém identificou a presença de pseudomicélios e blastoconídeos, sugestivos de Candida sp., hifas hialinas e septadas sugestivas de Aspergillus sp., e numerosos bacilos Gram negativos que em cultura foram identificados como de Serratia marcenses.

Foi feita a hipótese de aspergiloma pulmonar e iniciado tratamento com fluconazol 200 mg ao dia. Durante a internação a paciente evoluiu com hemoptise, necessitando de transfusão de concentrados de hemácias.

Devido aos repetidos episódios de hemoptise, optou-se pela abordagem cirúrgica. A paciente foi submetida a lobectomia esquerda, sendo retirado o lobo superior esquerdo e o ápice do lobo inferior (Figura 3). Decidiu-se pela abordagem em segundo tempo do pulmão direito.

À microscopia direta, foram visualizadas hifas sugestivas de Aspergillus sp. (Figura 4).

A paciente, no quarto dia, evoluiu com insuficiência respiratória, insuficiência renal aguda, choque séptico e evidenciou-se fístula broncopleural à esquerda. No sexto dia, ocorreu o óbito.

Na avaliação anatomopatológica, foi evidenciada a presença de hifas na cavidade pulmonar na coloração de Grocott. Encontraram-se ainda hifas e conídios no tecido pulmonar com infiltrado inflamatório e presença de células gigantes, sugerindo aspergilose invasiva (Figuras 5A a D).

Na cultura do material de biópsia, houve crescimento de $S$. marcenses, que dificultou o crescimento do fungo. Posteriormente, houve crescimento de Aspergillus flavus.

\section{Discussão}

A ocorrência de aspergilomas em pacientes soropositivos para HIV é uma preocupação antiga. Estudos da década passada já identificavam a ocorrência dessa doença nas cavidades de tuberculose e de Pneumocistis jirovecii, considerando a contagem de CD4 $<100$ células $/ \mathrm{mm}^{3}$ como fator de risco para a progressão da doença [Harkin et al, 1997]. 
Em nosso relato, a caverna secundária à tuberculose foi o fator predisponente ao para a aspergilose.

O sintoma mais comum é a hemoptise - a causa da internação da paciente - com incidência em torno de $80 \%$, sendo maciça em $30 \%$ dos casos com risco de morte [Shah et al, 2008]. As possíveis causas são a invasão dos vasos sanguíneos pelo Aspergillus sp.; a ação mecânica do patógeno nas paredes dos vasos; a presença de um importante suprimento sanguíneo para a cavidade a partir das artérias bronquial, axilar e subclávia; a elaboração de toxinas fúngicas; ou ainda pela lesão inflamatória ou antigênica [Mori et al, 2000].

À radiografia de tórax, pôde ser evidenciado o sinal do ar crescente (massa de densidade de partes moles rodeada por espaço de ar numa cavidade de paredes finas no lobo superior direito), o que pode ser verificado entre os exames de admissão da paciente. Embora seja considerado um sinal característico do micetoma, pode ser encontrado em neoplasias broncogênicas, cisto hidático e granulomatose de Wegener [Faruqi et al, 2009]. A radiografia em decúbito lateral pode demonstrar uma mudança da posição da massa dentro da cavitação, sugerindo aspergiloma [Panjabi et al, 2009].

A pesquisa do Aspergillus em escarro apresenta sensibilidade de 50\%, porém não é específica. De forma semelhante, anticorpos $\operatorname{IgG}$ séricos podem ser encontrados nos portadores, embora possuam baixa especificade, a não ser que altos títulos sejam evidenciados. Demonstraram sensibilidade de $78 \%$ no teste de imunodifusão [Babatasi et $a l, 2000]$. Em nosso relato, não foi evidenciado o patógeno no escarro, apenas no lavado bronco-alveolar e na microscopia direta do material da biópsia.

O tratamento de escolha é a cirurgia. A intervenção cirúrgica leva a maior sobrevida no seguimento de um ano, quando comparada à estratégia medicamentosa [Lee et al, 2009]. Entretanto, esse é um procedimento de alto risco. A principal indicação é a presença de hemoptises repetidas como no caso de nossa paciente.

O principal procedimento cirúrgico é a lobectomia. Contudo, outras técnicas como segmentectomia, pneumectomia, ressecção de parede e cavernoplastia podem ser indicadas a depender do número de aspergilomas e do grau de comprometimento do parênquima pulmonar [Park \& Jheon, 2002].

A inflamação gerada pelos aspergilomas complexos pode levar à densa fibrose com obliteração do espaço pleural, extensão para planos de dissecção extra-pleural e distorção de estruturas hílares, o que aumenta o risco da cirurgia [Shiraishi et al, 2006].

Possíveis complicações da cirurgia são empiema, sangramento, quilotórax (após lobectomia), infecção da ferida cirúrgica e fístula broncopleural ou, como no caso da paciente, em questão à insuficiência respiratória [Brik et al, 2008], numa série de 89 casos cirúrgicos, calcularam uma mortalidade de 5,7\% [Regnard et al, 2000]. Entretanto, essa taxa varia muito na literatura, a depender da complexidade do aspergiloma [Kim et al, 2005].

No caso de contra-indicação à cirurgia, por recusa do paciente ou má condição clínica como baixa reserva cardíaca e/ou pulmonar, pode-se utilizar o tratamento com imidazólicos.

O itraconazol oral é excretado no escarro e representa uma droga efetiva contra o Aspergilus sp. A dose deve ser de $200 \mathrm{mg}$ ou mais e o tempo de tratamento deve ser de no mínimo um ano. 
A instilação endobrônquica e intracavitária de vários agentes antifúngicos tem mostrado resultados promissores com o desaparecimento do micetoma. Entretanto, deve ser reservada para os casos inoperáveis. A eficácia do método depende do número e da extensão da drenagem brônquica, assim como a velocidade de degradação da bola fúngica. Não há consenso sobre a dose da instilação intracavitária de anfotericina B e o tempo de tratamento.

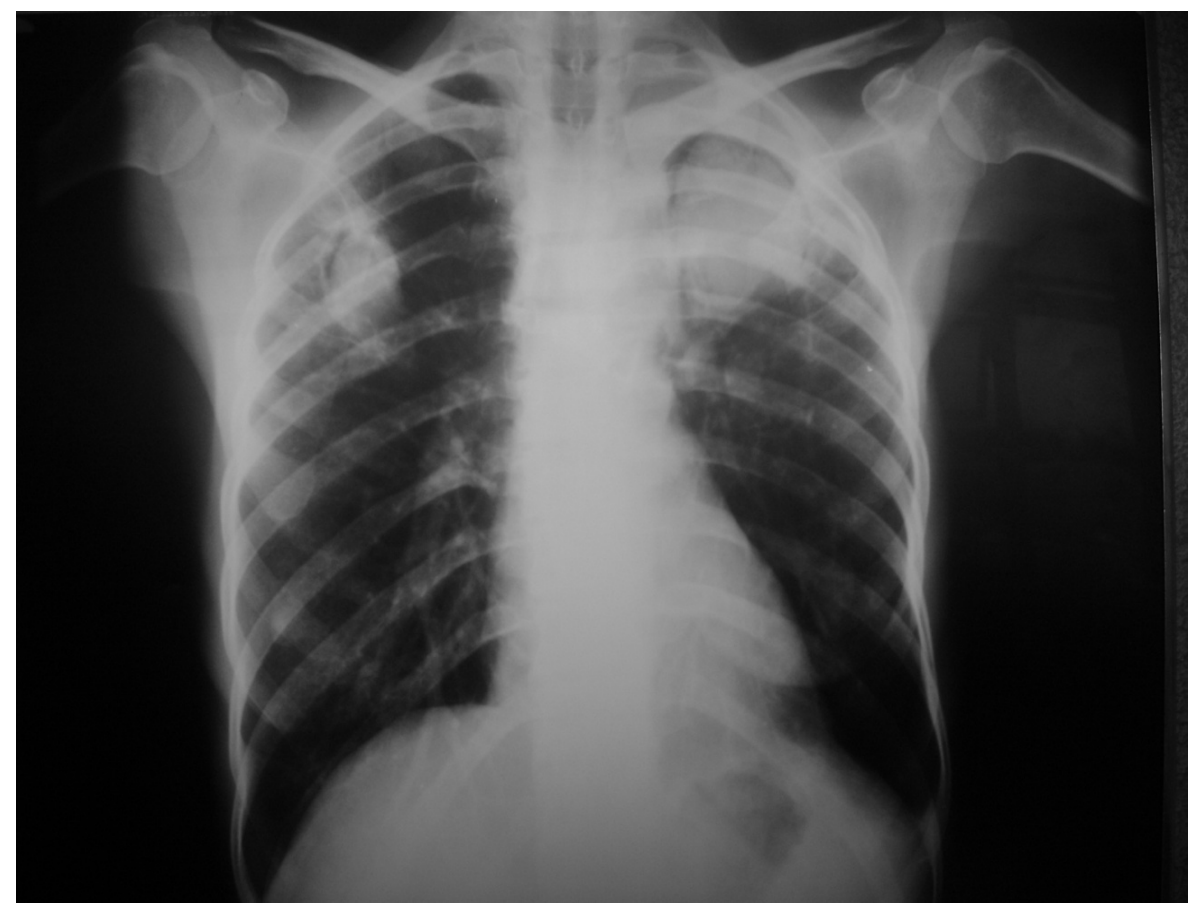

Figura 1. Radiografia de Tórax em PA mostrando duas áreas hiperdensas nos lobos superiores com ar ao redor delimitado por uma cavidade de paredes finas.

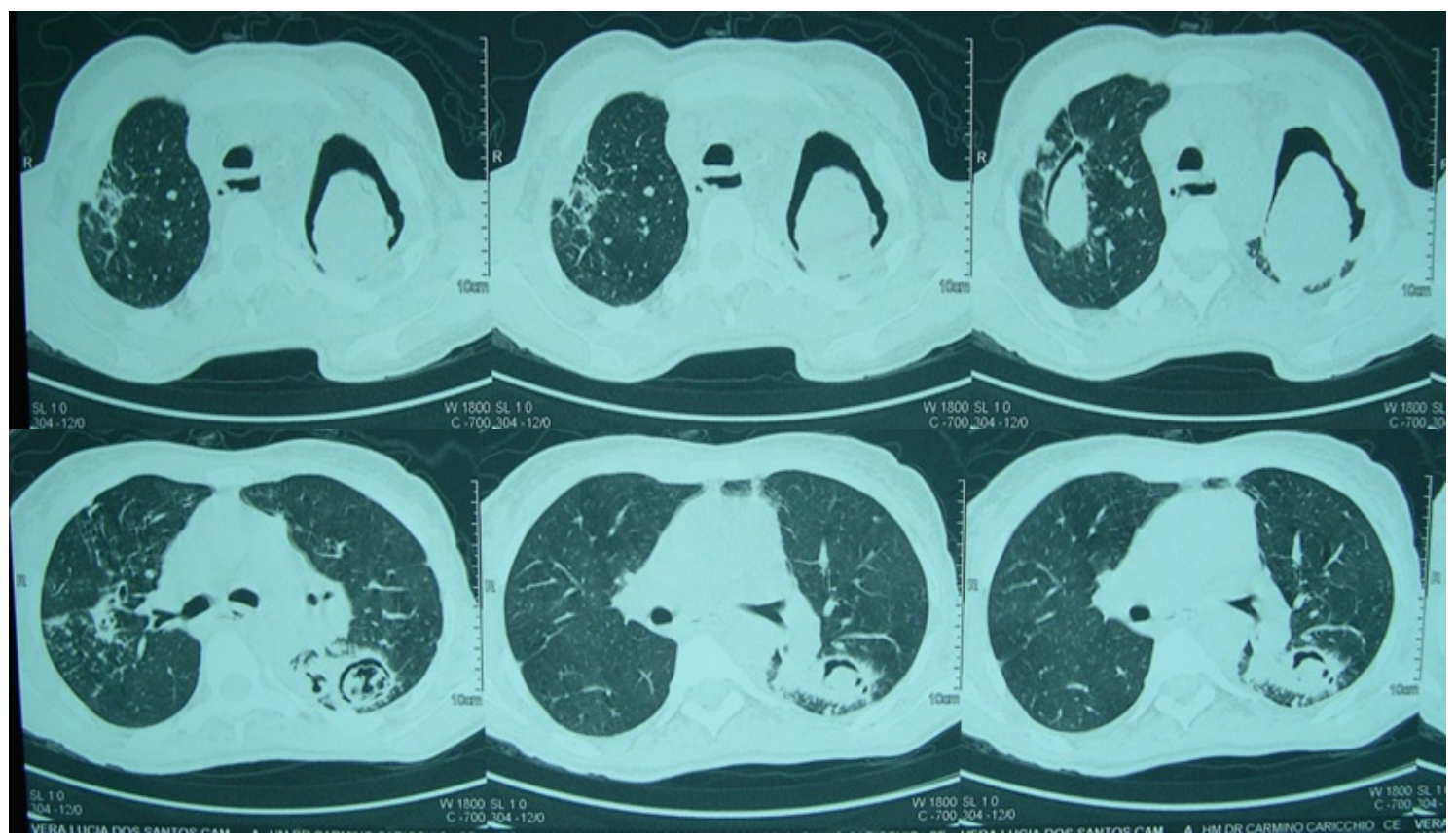

Figura 2. Tomografia de Tórax revelando cavidade pulmonar com presença de formação em seu interior de densidade de partes moles. 


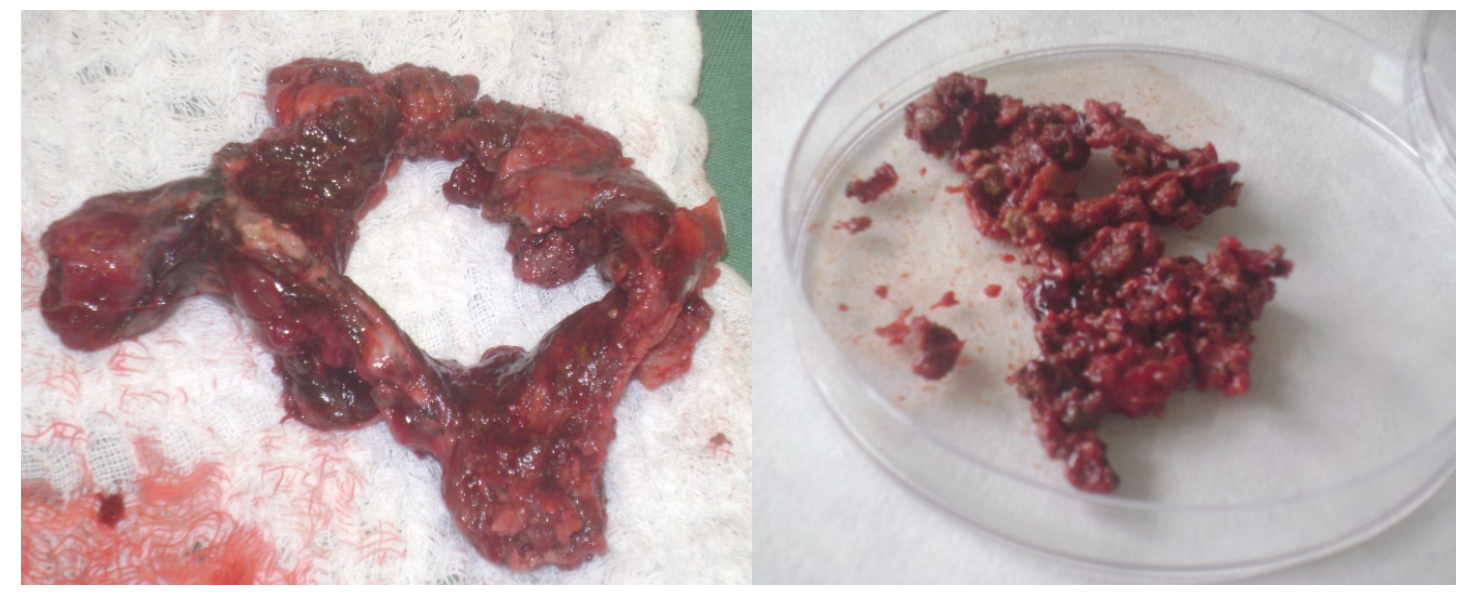

Figura 3A. Peça cirúrgica: Caverna pulmonar. 3B. Material friável retirado do interior da caverna.

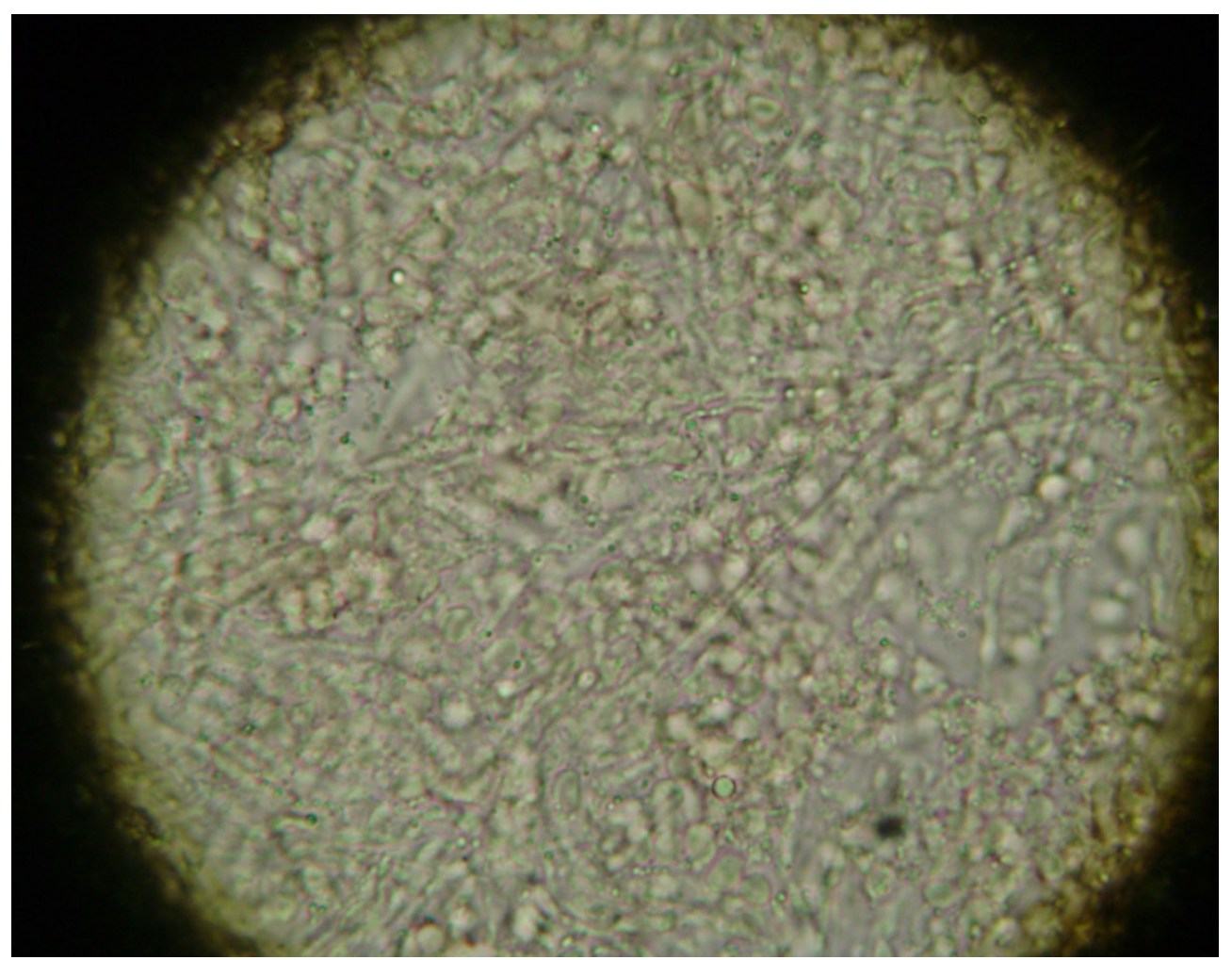

Figura 4. Esfregaço do material da biópsia retirado do interior da caverna em KOH, 1000X. 


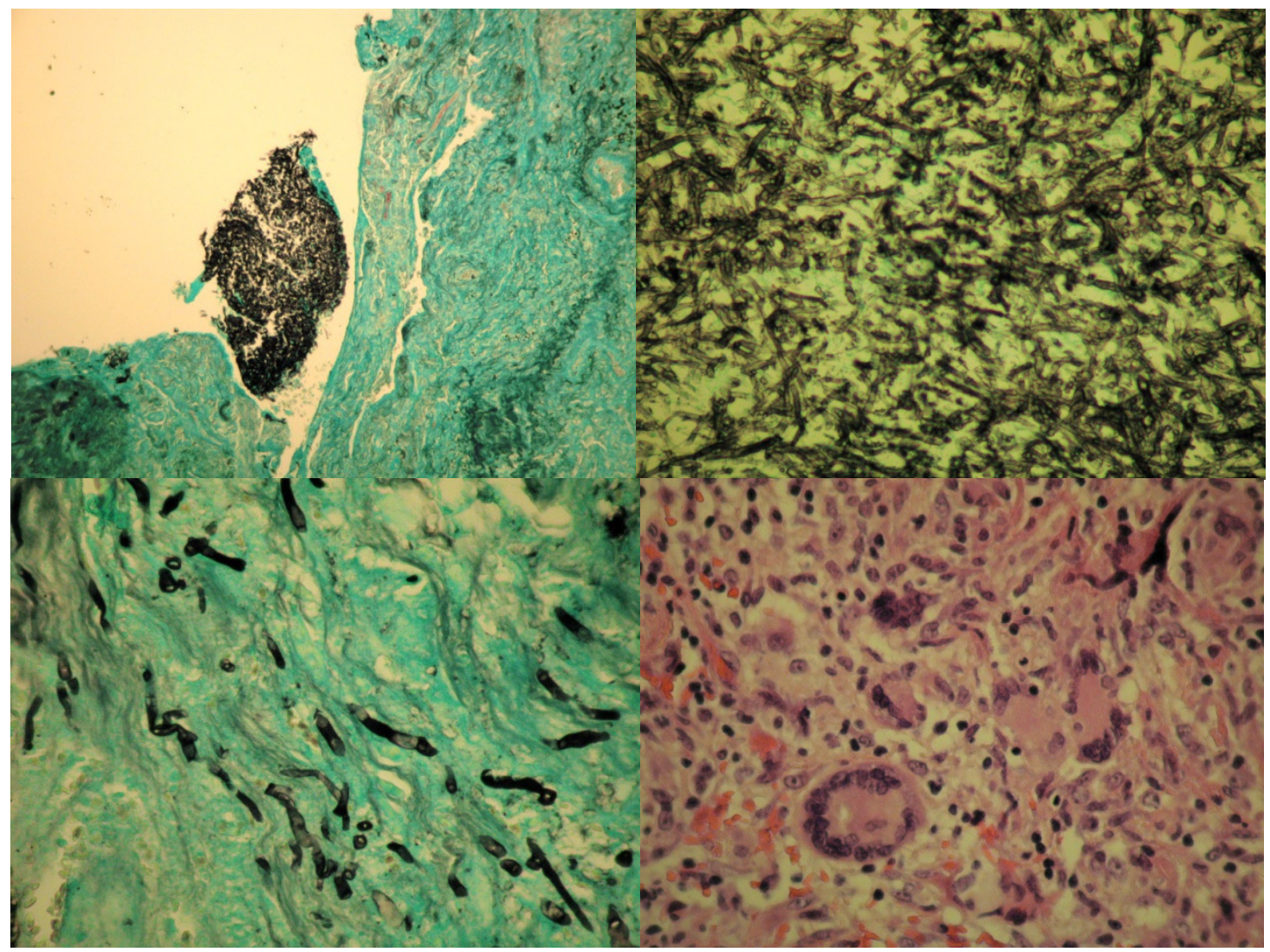

Figura 5A. Presença de hifas de Aspergillus sp. em cavidade. Coloração de Grocott. Aumento de 40x.; Figura 5B. Presença de hifas e conídios de Aspergillus sp. Coloração de Grocott. Aumento de 400x; Figura 5C: Tecido pulmonar com presença de hifas e conídios. Aspergilose invasiva. Coloração de Grocott, aumento de 400X; Figura 5D: Corte evidenciando presença de infiltrado inflamatório em tecido pulmonar com presença de células gigantes - Aspergilose invasiva. Coloração de Hematoxilina-Eosina. Aumento de 400X. 


\section{Referências}

Babatasi G, Massetti M, Chapelier A, et al. Surgical treatment of pulmonary aspergilloma: current outcome. J Thorac Cardiovasc Surg 2000;119: 906-12.

Baradkar VP, Mathur M, Kumar S. Uncomon presentation of pulmonary aspergilloma. Indian J Med Microbiol 2009; 27 (3): 270-2.

Brik A, Salem A, Kamal A, et al. Surgical outcome of pulmonary aspergilloma. Eur J Cardiothorac Surg 2008; (34): 882-5.

Faruqi S, Hughes D, Chauhan S, Wahbi Z. Aspergilloma. Intern Med J 2009; 39: 64-6.

Grima R, Krassas A, Bagan P, Badia A, Barthes F, Riquet M. Treatment of complicated pulmonary aspergillomas with cavernostomy and muscle flap: interest of concomitant limited thoracoplasty. Eur J Cardiothorac Surg 2009; 1-4.

Harkin TJ, McGuinness G, Naidich DP, Rom WN. Pulmonary aspergilloma and AIDS. A comparison of HIV-infected and HIV-negative individuals. Chest. 1997;111(3):612-8.

Kim YT, Kang MC, Sung SW, Kim JH. Good long-term outcomes after surgical treatment of simple and complex pulmonary aspergilloma. Ann Thorac Surg 2005; 79:294-8.

Lee JG, Lee CY, Park IK, et al. Pulmonary aspergilloma: Analysis of prognosis in relation to symptoms and treatment. J Thorac Cardiovasc Surg 2009; 138 (4): 820-5.

Mori $\mathrm{T}$, Ebe $\mathrm{T}$, Isonuma $\mathrm{H}$, et al. Aspergilloma: comparison of treatment methods and prognoses. J Infect Chemother. 2000; 6: 233-9.

Panjabi C, Sahay S, Shah A. Aspergilloma formation in cavitary sarcoidosis. J Bras Pneumol. 2009; 35(5):480-3.

Park CK, Jheon S. Results of surgical treatment for pulmonary aspergilloma. Eur $J$ Cardiothorac Surg 2002; 21: 918-23.

Regnard JF, Icard P, Nicolosi M, Spagiarri L, Magdeleinat P, Jauffret B et al. Aspergilloma: a series of 89 surgical cases. Ann Thorac Surg 2000; 69: 898-903.

Shah R, Vaideeswar P, Pandit S P. Pathology of pulmonary aspergillomas. Indian J Pathol Microbiol 2008; 51 (3): 342-5.

Shiraishi Y, Katsuragi N, Nakajima Y, Hashizume, Takahashi N, Miyasaka Y. Pneumonectomy for complex aspergilloma: is it still dangerous? Eur J Cardiothorac Surg 2006; 29: 9-13.

Soubani AO, Chandrasekar PH. The clinical spectrum of pulmonary aspergillosis. Chest 2002; 121: 1988-99. 